\title{
Identificação dos diagnósticos de enfermagem em recuperação pós-anestésica segundo a Teoria das necessidades humanas básicas
}

\author{
Identification of nursing diagnoses in post-anesthetic recovery according to the Theory \\ of basic human needs \\ Identificación de diagnósticos de enfermería en recuperación post-anestésica según la \\ Teoría de las necesidades humanas básicas
}

\section{Resumo}

Na sala de recuperação pós-anestésica são realizados cuidados intensivos em pacientes no pós-operatório imediato de procedimento anestésico-cirúrgico, permanecendo sob observação até o momento em que estejam conscientes, com reflexos protetores existentes e com os sinais vitais estáveis. De tal modo, o presente estudo visa identificar os diagnósticos de enfermagem mais frequentes em sala de recuperação pós-anestésica segundo a Teoria das necessidades humanas básicas. Trata-se de uma pesquisa de natureza quantitativa, do tipo descritivo com delineamento transversal, feita com um instrumento validado para a coleta dos dados, aplicado em pacientes maiores de 18 anos que passaram por atendimento cirúrgico, sendo admitidos na sala de recuperação pós-anestésica de uma unidade hospitalar de grande porte, referência em assistência de alta complexidade no Sistema Único de Saúde da região nordeste do Brasil. A amostra do estudo compôs-se de 48 pacientes, com trinta e duas mulheres $(66,7 \%)$ e dezesseis homens (33,3\%). Foram identificados 22 diagnósticos de Enfermagem, destes 17 corresponderam às necessidades humanas básicas psicobiológicas, 4 às necessidades humanas básicas psicossociais e 1 psicoespiritual. Diagnósticos encontrados em $100 \%$ da amostra: Risco de Infecção, Risco de Queda, Risco de Desequilíbrio da Temperatura Corporal, Risco de Aspiração, Integridade da Pele Prejudicada e Mobilidade no Leito Prejudicada. Em suma, os diagnósticos identificados são achados comuns em pacientes de pós-operatório imediato, sendo possível antecipar as ações e evitar que ocorram essas complicações e o agravamento da condição de saúde do paciente.

Palavras-chave: Centros cirúrgicos; Enfermagem perioperatória; Sala de recuperação; Diagnóstico de enfermagem.

\footnotetext{
Abstract

In the post-anesthetic recovery room, intensive care is performed on patients in the immediate postoperative period of anesthetic-surgical procedure, remaining under observation until they are conscious, with existing protective reflexes
} 
and with stable vital signs. In this way, the present study aims to identify the most frequent nursing diagnoses in a post-anesthetic recovery room according to the Theory of basic human needs. This is a quantitative research, of a descriptive type with a cross-sectional design, carried out with a validated instrument for data collection, applied to patients older than 18 years who underwent surgical care, being admitted to the post-anesthetic recovery room. Of a large hospital unit, a reference in highly complex assistance in the Unified Health System of the northeast region of Brazil. The study sample consisted of 48 patients, with thirty-two women $(66.7 \%)$ and sixteen men (33.3\%). 22 Nursing diagnoses were identified, of these 17 corresponded to basic psychobiological human needs, 4 to basic psychosocial human needs and 1 psycho-spiritual. Diagnoses found in 100\% of the sample: Risk of Infection, Risk of Fall, Risk of Body Temperature Imbalance, Risk of Aspiration, Impaired Skin Integrity and Impaired Bed Mobility. In short, the diagnoses identified are common findings in patients in the immediate postoperative period, making it possible to anticipate actions and prevent these complications from occurring and worsening the patient's health condition.

Keywords: Surgicenters; Perioperative nursing; Recovery room; Nursing diagnosis.

\section{Resumen}

En la sala de recuperación post-anestésica se realizan cuidados intensivos a los pacientes en el postoperatorio inmediato de procedimiento anestésico-quirúrgico, permaneciendo en observación hasta que estén conscientes, con reflejos protectores existentes y con signos vitales estables. De esta forma, el presente estudio tiene como objetivo identificar los diagnósticos de enfermería más frecuentes en una sala de recuperación post-anestésica según la Teoría de las necesidades humanas básicas. Se trata de una investigación cuantitativa, de tipo descriptivo con un diseño transversal, realizada con un instrumento validado para la recolección de datos, aplicada a pacientes mayores de 18 años que fueron intervenidos quirúrgicamente, siendo ingresados en la sala de recuperación post-anestésica. De una gran unidad hospitalaria, referente en asistencia de alta complejidad en el Sistema Único de Salud de la región noreste de Brasil. La muestra del estudio estuvo formada por 48 pacientes, con 32 mujeres $(66,7 \%)$ y 16 hombres $(33,3 \%)$. Se identificaron 22 diagnósticos de enfermería, de estos 17 correspondían a necesidades humanas psicobiológicas básicas, 4 a necesidades humanas psicosociales básicas y 1 psicoespiritual. Diagnósticos encontrados en el $100 \%$ de la muestra: riesgo de infección, riesgo de caída, riesgo de desequilibrio de la temperatura corporal, riesgo de aspiración, deterioro de la integridad de la piel y deterioro de la movilidad de la cama. En definitiva, los diagnósticos identificados son hallazgos comunes en los pacientes en el postoperatorio inmediato, lo que permite anticipar acciones y evitar que estas complicaciones ocurran y empeoren el estado de salud del paciente.

Palabras clave: Centros quirúrgicos; Enfermería perioperatoria; Sala de recuperación; Diagnóstico de enfermería.

\section{Introdução}

A Sistematização de Assistência de Enfermagem Perioperatória (SAEP) possibilita ao paciente uma assistência integralizada, complexa, segura e humanizada pela equipe de enfermagem. É entendida como um instrumento de cunho metodológico que sistematiza a atuação profissional e permite a compreensão, análise e prévia ideação dos resultados individuais às modificações de saúde (Ribeiro, Ferraz, \& Duran, 2017).

O perioperatório compreende os momentos pré-operatório, transoperatório e pós-operatório. O período pré-operatório imediato abrange as primeiras 24 horas anteriores à cirurgia e finda com o deslocamento do paciente ao centro cirúrgico (CC). Por sua vez, o período transoperatório tem início com o recebimento do paciente no centro cirúrgico e dura até sua saída da sala de cirurgia. O período pós-operatório engloba o momento após a execução da cirurgia. Pode ser dividido em três fases, sendo elas: recuperação anestésica, que tem início com a entrada do paciente na sala de recuperação pós-anestésica (SRPA) e vai até à liberação do mesmo para a unidade de origem; a fase de pós-operatório imediato (POI), que engloba as primeiras 24 horas após o procedimento cirúrgico; e por último o pós-operatório mediato, que inaugura depois das primeiras 24 horas após a cirurgia e estende até o momento da alta hospitalar (Prearo \& Fontes, 2019).

A sala de recuperação pós-anestésica apresenta rotinas peculiares no atendimento aos pacientes, sendo caracterizada por uma assistência crítica, que tem como meta a prestação de cuidados intensivos no pós-operatório imediato de procedimentos anestésico-cirúrgicos. O paciente deve permanecer na sala até que recupere a consciência e ocorra uma estabilização dos sinais vitais, com a finalidade de detectar e evitar complicações que possam ocorrer em consequência da anestesia ou do ato cirúrgico (Pereira et al., 2018).

A consulta de enfermagem evidencia-se como uma prática de elevada importância para realização da assistência do 
enfermeiro, para o método de ensino-aprendizagem e no fomento à elaboração e fortalecimento do conhecimento científico em enfermagem. É um ato de cunho privativo do Enfermeiro e possibilita um cuidado que extrapola os fundamentos biológicos e permite uma visão social da situação (Crivelaro, Posso, Gomes, \& Papini, 2020).

Sendo esta, extremamente crucial para o reconhecimento da enfermagem como ciência. Apresentando-se como um conjunto de ações organizada e sistematizadas relacionadas com a intenção de prestar o cuidado ao ser humano, caracterizada pelo vínculo e dinamismo entre suas etapas (Crivelaro et al., 2020).

Neste tocante, objetiva-se que a identificação desses diagnósticos possam contribuir para o cuidado de Enfermagem aos pacientes que passarem pela sala de recuperação pós-anestésica, por meio do reconhecimento de problemas e potencialidades mais comuns, que possam direcionar os profissionais de Enfermagem para uma melhor prática clínica e seu aperfeiçoamento no cuidado diário aos pacientes.

\section{Metodologia}

Trata-se de um estudo de natureza quantitativa, do tipo descritivo com delineamento transversal. Nos métodos de natureza quantitativa, a coleta de dados é feita por meio do uso de medições de grandezas e obtém-se por meio da metrologia, números com suas respectivas unidades; gerando conjuntos ou massas de dados que podem ser analisados através de técnicas matemáticas como é o caso das porcentagens, estatísticas e probabilidades, métodos numéricos e analíticos, geração de equações e/ou fórmulas matemáticas aplicáveis a algum processo (Pereira, Shitsuka, Parreira, \& Shitsuka, 2018).

A pesquisa de campo foi realizada na sala de recuperação pós-anestésica de um hospital público de grande porte, voltado para ensino e pesquisa, referência em assistência de alta complexidade no Sistema Único de Saúde (SUS) do Nordeste. Essa unidade possui seis leitos de recuperação pós-anestésica e supre a demanda de sete salas cirúrgicas realizando, em média, 400 cirurgias por mês. O estudo foi dividido em duas etapas: a primeira voltada aos dados de caracterização da amostra e a segunda consistindo no exame físico, sendo este e a anamnese subsídios à identificação dos diagnósticos de enfermagem.

Os sujeitos da pesquisa foram selecionados a partir da identificação dos pacientes admitidos no centro cirúrgico que tinham indicação de ir para a sala de recuperação pós-anestésica. Inicialmente, ocorreu a aproximação no período transoperatório, no momento da admissão no Centro Cirúrgico, por meio da aceitação em participar do estudo, após esclarecido o processo da coleta e assinatura do Termo de Consentimento Livre e Esclarecido (TCLE).

A amostra foi determinada por meio do seguinte critério de inclusão: pacientes maiores de 18 anos que foram para a sala de recuperação pós-anestésica; e como critério de exclusão: cirurgias oftalmológicas, por tratar-se de pacientes externos e, que muitas vezes, recebem alta logo após o procedimento, além de apresentarem diagnósticos específicos.

Os dados foram coletados através de um instrumento previamente estruturado (Cunha \& Peniche, 2007), sendo utilizadas cinco seções deste: 1. Identificação inicial: idade, sexo, cirurgia realizada, tipo de anestesia, condição prévia do paciente (alergias e antecedentes clínicos); 2. Controle do estado clínico do paciente na sala de recuperação pós-anestésica: frequência cardíaca e respiratória, temperatura, pulso, pressão arterial sistêmica, saturação de oxigênio e nível de dor; 3. Índice de Aldrete e Kroulik para pacientes adultos; 4. Exame físico de acordo com os sistemas do corpo humano (respiratório, neurológico, cardiovascular, digestório, renal e tegumentar) e avaliação da incisão cirúrgica (quanto ao aspecto e local do curativo); 5. Prescrição e evolução de enfermagem.

Para tanto, as variáveis do estudo são: gênero, faixa etária, doenças crônicas, procedimentos anestésicos e cirúrgicos. O projeto teve aprovação do Comitê de Ética em Pesquisa da Instituição sob o número CAAE 02843818.3.0000.5013, respeitando os princípios e diretrizes contidos na Resolução CNS nº 466/12 do Conselho Nacional de Pesquisa em Saúde que envolve seres humanos. 


\section{Resultados e Discussão}

O centro cirúrgico é considerado uma das unidades mais importantes e específicas do setor hospitalar, no qual são compartilhados inúmeros processos e subprocessos, ligados direta ou indiretamente à produção de cirurgias. Em circunstâncias assépticas ideais, o CC tem por objetivo promover segurança do paciente, além de estrutura para a equipe que o assiste (Amaral, Spiri, \& Bocchi, 2017).

Os profissionais que atuam nesse local devem estar aptos a enfrentar as exigências impostas pelo ambiente, favorecendo à qualidade da assistência e bem-estar do paciente. O enfermeiro encontra-se a frente desta unidade e é peça fundamental para que a equipe realize um bom trabalho, tornando-se líder e coordenador das atividades (Souza et al., 2019).

$\mathrm{O}$ referido hospital realiza cirurgias de alta complexidade e, no período destinado à coleta de dados, sendo este de onze dias distribuídos em três meses, a contar de janeiro a março de 2020, foram atendidos em média 375 pacientes por mês nesse Centro Cirúrgico. Para análise dos dados e caracterização da amostra, foi feita uma tabulação referente a faixa etária de 48 pacientes (100\%) que passaram por algum procedimento cirúrgico e permaneceram na sala de recuperação pós-anestésica.

A amostra compôs-se de trinta e dois pacientes do sexo feminino (66,7\%) e dezesseis do sexo masculino (33,3\%). É possível verificar, no Quadro 1, que a maior parte é composta por adultos de 48 aos 57 anos, sendo doze pacientes (25\%), seguido do grupo com idades de 38 aos 47 anos, com nove pacientes $(18,8 \%)$ e do grupo de 58 aos 67 anos, representando um total de oito pacientes $(16,7 \%)$.

Quadro 1 - Caracterização da amostra por faixa etária.

\begin{tabular}{|c|c|c|}
\hline Idade & Número $(\mathbf{N}$ total = 48) & Percentagem (\%) \\
\hline $18-27$ anos & 4 & $8,3 \%$ \\
\hline $28-37$ anos & 5 & $10,4 \%$ \\
\hline $38-47$ anos & 9 & $18,8 \%$ \\
\hline $48-57$ anos & 12 & $25 \%$ \\
\hline $58-67$ anos & 8 & $16,7 \%$ \\
\hline $68-77$ anos & 6 & $8,3 \%$ \\
\hline $78-87$ anos & 4 & $100 \%$ \\
\hline TOTAL & 48 & $12,5 \%$ \\
\hline
\end{tabular}

Fonte: Autores (2021).

Macedo (2020), em seu estudo a respeito da análise do grau de dependência de cuidados de enfermagem em uma unidade de recuperação pós-anestésica, realizado em um hospital de grande porte de Pernambuco, teve a maior parte da sua amostra composta pela população de 60 anos ou mais, sendo representada por vinte e quatro pacientes de um total de setenta $(34,28 \%)$. Esse fato deve-se ao próprio processo do envelhecimento que está associado a maior prevalência de comorbidades, os pacientes mais idosos tendem a apresentar mais eventos mórbidos. Idade avançada representa uma condição de vulnerabilidade, assim como doenças crônicas, fragilidade e a natureza invasiva do procedimento cirúrgico são outros importantes preditores de mortalidade (Silva, Casimiro, Oliveira, Ferreira, \& Abelha, 2020).

No que se refere às doenças crônicas, vinte e sete pacientes negaram comorbidades $(56,3 \%)$, sete pacientes eram hipertensos e diabéticos (14,6\%), seis apresentavam apenas hipertensão arterial sistêmica (12,5\%), dois com diabetes mellitus $(4,2 \%)$, um paciente cardiopata e hipertenso $(2,1 \%)$ e um registrando distúrbios neurológicos $(2,1 \%)$. Os quatro $(8,3 \%)$ 
pacientes com outras doenças crônicas apresentaram trombose venosa profunda (TVP), etilismo e/ou tabagismo.

As comorbidades clínicas constituem um importante fator de risco para maior mortalidade, além de estarem associadas ao surgimento de complicações pós-cirúrgicas, que podem ser imediatas ou tardias. A avaliação pré-operatória, portanto, deve estar baseada em exame criterioso de toda história clínica do paciente, de modo a estabelecer medidas de prevenção de eventos mórbidos, evitar iatrogenias, definir critérios que alertem para um maior risco e desenvolver enfoque multidisciplinar (Edelmuth, Sorio, Sprovieri, Gali, \& Peron, 2018).

Após a caracterização da amostra, foi realizada a tabulação dos Diagnósticos de Enfermagem identificados de acordo com a classificação internacional para a prática de Enfermagem conforme o Quadro 2.

Quadro 2 - Diagnósticos de Enfermagem identificados no pós-operatório imediato de cirurgias, durante a permanência do paciente na sala de recuperação pós-anestésica, segundo Teoria das necessidades humanas básicas.

\begin{tabular}{|c|c|c|c|c|}
\hline $\begin{array}{l}\text { Necessidades Humanas } \\
\text { Básicas }\end{array}$ & Subgrupo & Diagnóstico de Enfermagem & Número & Percentagem \\
\hline \multirow{17}{*}{ PSICOBIOLÓGICAS } & \multirow{4}{*}{ Ambiente } & Risco de Infecção & 48 & $100 \%$ \\
\hline & & Risco de Queda & 48 & $100 \%$ \\
\hline & & $\begin{array}{c}\text { Risco de Desequilíbrio } \\
\text { da Temperatura } \\
\text { Corpora }\end{array}$ & 48 & $100 \%$ \\
\hline & & Risco de Aspiração & 48 & $100 \%$ \\
\hline & $\begin{array}{l}\text { Integridade Cutâneo- } \\
\text { Mucosa }\end{array}$ & Integridade da Pele Prejudicada & 48 & $100 \%$ \\
\hline & $\begin{array}{l}\text { Locomoção/ } \\
\text { Motilidade }\end{array}$ & Mobilidade no Leito Prejudicada & 48 & $100 \%$ \\
\hline & \multirow{2}{*}{ Regulação Térmica } & Hipotermia & 45 & $93,7 \%$ \\
\hline & & Tremor/Calafrio & 3 & $6,2 \%$ \\
\hline & \multirow[b]{2}{*}{ Regulação Vascular } & Pressão Arterial Diminuída & 4 & $8,3 \%$ \\
\hline & & Pressão Arterial Aumentada & 1 & $2,1 \%$ \\
\hline & $\begin{array}{l}\text { Regulação } \\
\text { Neurológica }\end{array}$ & Estado de Alerta Prejudicado & 39 & $81,2 \%$ \\
\hline & Eliminação & Náusea & 19 & $39,6 \%$ \\
\hline & $\begin{array}{l}\text { Percepção dos Órgãos } \\
\text { dos Sentidos }\end{array}$ & Dor Aguda & 14 & $29,2 \%$ \\
\hline & \multirow{4}{*}{ Oxigenação } & Frequência Respiratória Diminuída & 13 & $27,1 \%$ \\
\hline & & Frequência Cardíaca Diminuída & 5 & $10,4 \%$ \\
\hline & & Hipoxemia & 5 & $10,4 \%$ \\
\hline & & Processo de Transpiração Prejudicado & 1 & $2,1 \%$ \\
\hline \multirow{4}{*}{ PSICOSSOCIAIS } & \multirow{4}{*}{$\begin{array}{l}\text { Segurança } \\
\text { Emocional }\end{array}$} & Ansiedade & 29 & $60,4 \%$ \\
\hline & & $\begin{array}{l}\text { Risco de Síndrome do Estresse por } \\
\text { Mudança }\end{array}$ & 5 & $10,4 \%$ \\
\hline & & Medo & 3 & $6,2 \%$ \\
\hline & & Risco de Solidão & 1 & $2,1 \%$ \\
\hline PSICOESPIRITUAIS & $\begin{array}{l}\text { Religiosidade/ } \\
\text { Espiritualidade }\end{array}$ & Angústia Espiritual & 5 & $10,4 \%$ \\
\hline
\end{tabular}

Fonte: Autores (2021). 
Foram identificados seis diagnósticos de risco durante a permanência dos pacientes na SRPA. Quatro deles apresentaram incidência de $100 \%$ e estavam, portanto, presentes em todos os quarenta e oito pacientes da pesquisa: Risco de Infecção, Risco de Queda, Risco de Desequilíbrio da Temperatura Corporal e Risco de Aspiração; já o Risco de Síndrome do Estresse por Mudança esteve presente em cinco pacientes, correspondendo a 10,4\% e o diagnóstico de Risco de Solidão presente em um paciente $(2,1 \%)$. Pode-se verificar que foram identificados dezesseis diagnósticos reais, dos quais dois estiveram presentes em todos os pacientes da amostra: Integridade da Pele Prejudicada e Mobilidade no Leito Prejudicada. Os diagnósticos: Hipotermia, Estado de Alerta Prejudicado, Dor Aguda, Ansiedade, Náusea, Frequência Respiratória Diminuída, Frequência Cardíaca Diminuída, Hipoxemia e Angústia Espiritual, estiveram presentes em percentagens maiores que 10\%, tendo uma representatividade de no mínimo cinco pacientes em um total de quarenta e oito (100\%).

Os diagnósticos foram divididos de acordo com as Necessidades Humanas Básicas (NHB) de Wanda de Aguiar Horta, nas categorias de psicobiológicas, psicoespirituais e psicossociais, separadas em subgrupos de ambiente, integridade cutâneomucosa, locomoção/motilidade, regulação térmica, regulação vascular, regulação neurológica, eliminação, percepção dos órgãos dos sentidos e oxigenação em psicobiológicas; segurança emocional em psicoespirituais e religiosidade/espiritualidade em psicossociais.

A Teoria de Wanda Horta é baseada em três leis gerais que regem os fenômenos universais, sendo: a lei do equilíbrio, onde todo o universo se mantém por processos de equilíbrio dinâmico; a lei da adaptação, que todos os seres do universo interagem com seu meio externo buscando sempre formas de ajustamento para permanecerem em equilíbrio; a lei do holismo, tendo o universo, o ser humano e a célula como um todo, e esse todo não é simplesmente soma das partes constituintes de cada ser. A inter-relação dessas leis estabelece a amálgama que constitui a teoria (Ubaldo, Matos, \& Salum, 2015).

Para tanto, fundamenta-se na Teoria de Motivação Humana de Maslow que tem sua essência na hierarquização das Necessidades Humanas Básicas (NHB). Essas estão dispostas em cinco níveis de prioridades, traçados do mais básico para o mais complexo, a saber: necessidades fisiológicas, segurança e proteção, amor e gregarismo, autoestima e autorrealização. Na Enfermagem, emprega-se a proposição de João Mohana que classifica a NHB em: psicobiológica, psicossocial e psicoespiritual (Marinho, Oliveira, Silva, Oliveira, \& Leite, 2020).

O paciente em POI, localizado na SRPA, está suscetível às complicações do aparelho termorregulador, sensóriomotor, cardiorrespiratório, tegumentar, urinário, gastrointestinal e imunológico, além do estado emocional. Essas manifestações orgânicas dependem de fatores considerados intrínsecos e extrínsecos ao paciente. De forma intrínseca pode-se citar a extensão e procedimento cirúrgico realizado, e as extrínsecas como inspeção rotineira de aparelhos e melhoria de recursos humanos, por exemplo (Santo et al., 2020).

Torna-se, portanto, imprescindível que o profissional de Enfermagem que atua em SRPA apresente conhecimento sobre fisiologia, métodos cirúrgicos, fundamentos da farmacodinâmica anestésica e analgésica. É importante estar preparado para todas as intercorrências que possam vir a surgir do estresse cirúrgico que podem comprometer a homeostase orgânica (Prearo \& Fontes, 2019).

Ao conhecer as complicações que podem existir no período pós-operatório imediato, é possível desenvolver um raciocínio clínico em torno da situação estabelecida, na qual as situações de emergências são previstas (Souza, Silva, \& Bassine, 2020).

A assistência de enfermagem perioperatória, voltada à satisfação das necessidades dos indivíduos na fase anestésicocirúrgica, necessita de uma abordagem reflexiva quanto ao seu processo de ensino. De modo a fazer com que a mesma seja indispensável para o profissional que trabalha na área perioperatória. Os enfermeiros que atuam nesse setor devem estar capacitados e treinados a atuar de maneira individual, atendendo o paciente em suas necessidades; além de fundamentar suas ações na aplicação criativa desses conhecimentos e ter habilidades e competências que visem um cuidado individualizado ao 
paciente cirúrgico (Alvarez, Girondi, \& Knihs, 2018).

\section{Conclusão}

O estudo foi realizado com quarenta e oito pacientes da sala de recuperação pós-anestésica, a maioria do gênero feminino, com faixa etária predominante dos 38 aos 57 anos; sendo identificados, de acordo com a Teoria das necessidades humanas básicas de Wanda Horta, vinte e dois diagnósticos de Enfermagem, dos quais dezessete são classificados para as necessidades humanas básicas psicobiológicas, quatro para as necessidades humanas básicas psicossociais e um para psicoespiritual.

Os principais achados da pesquisa apontam a predominância de Diagnósticos de Risco, como: Risco de Infecção, Risco de Queda, Risco de Desequilíbrio da Temperatura Corporal, Risco de Aspiração; e dois Diagnósticos Reais: Integridade da Pele Prejudicada e Mobilidade no Leito Prejudicada, que tiveram incidência de $100 \%$ dos pacientes que compuseram a amostra. Reforçando, dessa forma, a importância desse trabalho para a identificação dos Diagnósticos de Enfermagem mais recorrentes, de modo a anteceder as ações e evitar que ocorram essas complicações e o agravamento da condição de saúde do paciente.

Por tratar-se de um estudo que dá suporte à boa atuação da equipe de enfermagem no centro cirúrgico é fundamental a continuidade deste para o favorecimento da implantação da SAEP, comprovando a eficiência da Sistematização da Assistência de Enfermagem Perioperatória que, por sua vez, irá colaborar para o desenvolvimento da evolução do estado clínico dos pacientes, evitando desgastes futuros causados pelo estresse profissional e o risco de uma assistência inadequada; gerando assim, benefícios para instituição, equipe e, principalmente, para o paciente. Dessa forma, os resultados apresentados sustentam a importância da realização de pesquisas futuras robustas para ampliar e aprofundar as evidências científicas que envolvem essa temática.

\section{Referências}

Alvarez, A. G., Girondi, J. B. R., \& Knihs, N. S. (2018). Metodologias Ativas na Educação em Enfermagem Perioperatória. Revista SOBECC, 23 (1), 1-2. $10.5327 / Z 1414-4425201800010001$.

Amaral, J. A. B., Spiri, W. C., \& Bocchi, S. C. M. (2017). Indicadores de Qualidade em Enfermagem com Ênfase no Centro Cirúrgico: Revisão Integrativa da Literatura. Revista SOBECC, 22 (1), 42-51. 10.5327/Z1414-4425201700010008.

Crivelaro, P. M. S., Posso, M. B. S., Gomes, P. C., \& Papini, S. J. (2020). Consulta de Enfermagem: Uma Ferramenta de Cuidado Integral na Atenção Primária à Saúde. Brazilian Journal of Development, 6 (7), 49310-49321. 10.34117/bjdv6n7-542.

Cunha, A. L. S. M., \& Peniche, A. C. G. (2007). Validação de um Instrumento de Registro para Sala de Recuperação Pós-Anestésica. Acta Paulista de Enfermagem, 20 (2), 151-160. http://dx.doi.org/10.1590/S0103-21002007000200007.

Edelmuth, S. V. C. L., Sorio, G. N., Sprovieri, F. A. A, Gali, J. C., \& Peron, S. F. (2018). Comorbidades, Intercorrências Clínicas e Fatores Associados à Mortalidade em Pacientes Idosos Internados por Fratura de Quadril. Revista Brasileira de Ortopedia, 53 (5), 543-551. http://dx.doi.org/10.1016/j.rboe.2018.07.014.

Macedo, J. K. S. S. (2020). Análisis del Grado de Dependencia de los Cuidados de Enfermería en una Unidad de Recuperación Post Anestesia. Enfermería Actual de Costa Rica, n. 38. 10.15517/revenf.v0i38.38332.

Marinho, C. L. A., Oliveira, A. S., Silva, R. S., Oliveira, J. F., \& Leite, A. M. C. (2020). Necessidades Humanas Básicas de Pessoas em Hemodiálise sob à Luz da Teoria de Wanda Horta. Ciência, Cuidado e Saúde, 19:e47832, 1-7. 10.4025/cienccuidsaude.v19i0.47832.

Pereira, A. S., Shitsuka, D. M., Parreira, F. J., \& Shitsuka, R. (2018). Metodologia da Pesquisa Científica. UFSM.: https://repositorio.ufsm.br/bitstream/handle/1/15824/Lic_Computacao_Metodologia-Pesquisa-Cientifica.pdf?sequence=1 .

Pereira, E. B. F., Lima, G. P., Silva, H. A. G. B., Teixeira, K. M. H., Modesto, B. C. M., \& Novaes, M. A. (2018). Avaliação da Qualidade dos Registros de Enfermagem nos Cuidados Pós-Operatórios Imediatos. Revista SOBECC, 23 (1), 21-27. 10.5327/Z1414-4425201800010005.

Prearo, M., \& Fontes, C. M. B. (2019). Sistematização da Assistência de Enfermagem na Sala de Recuperação Pós-Anestésica: Revisão Integrativa. Enfermagem em Foco, 10 (7), 135-140. Obtido de: https://pesquisa.bvsalud.org/portal/resource/pt/biblio-1051469. 
Research, Society and Development, v. 10, n. 3, e20210313201, 2021

(CC BY 4.0) | ISSN 2525-3409 | DOI: http://dx.doi.org/10.33448/rsd-v10i3.13201

Ribeiro, E., Ferraz, K. M. C. F., \& Duran, E. C. M. (2017). Atitudes dos Enfermeiros de Centro Cirúrgico Diante da Sistematização da Assistência de Enfermagem Perioperatória. Revista SOBECC, 22 (4), 201-207. 10.5327/Z1414-4425201700040005.

Santo, I. M. B. E., Matos, J. C., Silva, C. J., Almeida, R. P., Santos, J. L. P., Silva, S. M., Caetano, E. R., Lima, R. D., Nunes, K. S., Barbosa, S. S. (2020). Perioperative Nursing Care Systematization: A Reflex of Applicability in the Care Process. Electronic Journal Collection Health, 43:e2945, 1-8. https://doi.org/10.25248/reas.e2945.2020.

Silva, D. J. N., Casimiro, L. G. G., Oliveira, M. I. S., Ferreira, L. B. C., Abelha, F. J. P. A. (2020). The very Elderly Surgical Population in a Critically Illscenario: Clinical Characteristics and Outcomes. Revista Brasileira de Anestesiologia, 70 (1), 3-8. https://doi.org/10.1016/j.bjane.2020.02.002.

Souza, C. D. M., Silva, A. A., \& Bassine, C. P. J. (2020). The Importance of Nursing Team in Postanesthetic Recovery. Revista da Faculdade Sant' Ana, 4 (1), 4-13. Obtido de: https://www.iessa.edu.br/revista/index.php/fsr/article/view/1623.

Souza, I. B., Tenório, H. A. A., Gomes Junior, E. L., Sarmento Neto, M. L., Almeida, B. R., \& Marques, E. S. (2019). Percepção do Cliente no Perioperatório sobre o Cuidado de Enfermagem no Centro Cirúrgico. Revista de Enfermagem e Atenção à Saúde, 26:e860, 1-8. https://doi.org/10.25248/reas.e840.2019.

Ubaldo, I. Matos, E. Salum, N. C. (2015). Diagnósticos de Enfermagem da Nanda-I com Base nos Problemas Segundo Teoria de Wanda Horta. Cogitare Enfermagem, 20 (4), 687-694. Obtido de: https://revistas.ufpr.br/cogitare/article/view/40468/26628. 